\title{
Range of hip abduction after preventive and reconstructive surgery in cerebral palsy: a longitudinal registry study of 307 children
}

\author{
Gunnar HÄGGLUND ${ }^{1,2}$ and Philippe WAGNER ${ }^{1,3}$
}

\author{
${ }^{1}$ Lund University, Department of Clinical Sciences, Lund, Orthopedics; ${ }^{2}$ Department of Orthopedics, Skane University Hospital, Lund; ${ }^{3}$ Centre for Clinical \\ Research, Uppsala University, Region Västmanland, Västerås, Sweden \\ Correspondence: gunnar.hagglund@med.lu.se \\ Submitted 2021-06-25. Accepted 2021-09-30.
}

Background and purpose - Hip dislocation in cerebral palsy (CP) is caused by altered muscle forces on the joint during typical hip positioning in adduction-flexion-inward rotation. Preventive surgery includes adductor-psoas lengthening (APL) or varus derotation osteotomy (VDRO) of the proximal femur. We assessed the changes in the hip abduction range after these operations.

Patients and methods - Data were obtained from the Swedish Surveillance Programme for CP. The range of hip abduction before and up to 18-36 months after surgery was assessed for all children who underwent APL or VDRO. Data for 1 hip per child was assessed. Ordinary linear regression was used.

Results - In the 150 children who underwent APL, the mean range of abduction increased from $29^{\circ}$ (95\% confidence interval $[\mathrm{CI}] 28-32$ ) preoperatively to $37^{\circ}$ (CI 35-39) at 18-36 months. In the 157 children who underwent VDRO, the respective mean values were $30^{\circ}$ (CI 29-32) and $29^{\circ}$ (CI 28-31). The mean difference in preoperative abduction between sides was greater in children who underwent unilateral $\left(9.4^{\circ}\right.$, CI 7.8-11) than bilateral (5.5 , CI 3.4-7.6) VDRO. At 18-36 months postoperatively, the differences between sides were almost unchanged.

Interpretation - The range of hip abduction increased after APL but remained unchanged after VDRO. This may explain the normal development of hip displacement after these operations. Differences in abduction between sides were not substantially affected by whether VDRO was performed uni- or bilaterally.
Hip dislocation in cerebral palsy $(\mathrm{CP})$ is caused by altered muscle forces on the hip joint. The stronger and more spastic adductor and flexor muscles shift the force vector from the anteromedial to posterolateral direction during hip positioning in adduction, flexion, and inward rotation (1).

The passive range of hip abduction normally decreases with age in children with CP (2). A spastic muscle will not allow stretch to the same degree as a muscle with normal tone. As a consequence, spasticity may inhibit growth in the length of the muscle, which results in the development of muscle contracture as measured by a decreasing range of joint motion (3). There are 2 principal surgical methods to treat hip dislocation in CP: preventive and reconstructive surgery. Lengthening of the adductor and iliopsoas muscles or tendons (APL) is a preventive surgery that aims to reduce the muscle imbalance and increase the capacity for abduction. There is a consensus that this operation should be performed bilaterally to prevent later contralateral dislocation (4-6).

Proximal femoral varus derotation osteotomy (VDRO), sometimes combined with pelvic osteotomy, is a reconstructive surgery that aims to correct the displacement momentarily. This varization can reduce the range of abduction and, to compensate for this, VDRO is often combined with APL and shortening of the femur. Opinions differ as to whether VDRO should be performed bilaterally, even in patients with unilateral hip displacement (7-9). After both APL and VDRO, there is a risk of recurrent hip displacement and the need for repeated surgery (10-12).

The Swedish Surveillance Programme for CP (CPUP) is a follow-up program and register for individuals with CP. CPUP started in southern Sweden (1.2 million inhabitants) in 1994 and, since 2006 , has included $>95 \%$ of all children and adolescents with CP in Sweden (10.4 million inhabitants) born 
in 2000 and later (13). In CPUP, the hips are examined with repeated measurements of passive range of hip motion. We used CPUP data to compare the development of the range of hip abduction after APL and VDRO. We compared the individual differences in abduction between sides between children operated on unilaterally and bilaterally.

\section{Patients and methods}

This was a longitudinal register-based study that used data from CPUP. In CPUP, children are examined by their local physiotherapist at intervals based on age and gross motor function according to the Gross Motor Function Classification Scale (GMFCS), a 5-point scale in which level V indicates the greatest disability (14). Children in levels II-V are examined twice a year until age 6 years and thereafter annually until age 18 years. The examination includes measurement of hip abduction with a goniometer according to a manual that describes the positions of the child and the goniometer arms. Hip abduction is measured in the supine position with the hips extended and knees flexed. The stationary arm is placed along a line joining the 2 anterior superior iliac spines, and the movable arm is placed parallel to the longitudinal axis of the femur. The abduction of the hip is done slowly (2).

In Sweden, surgical procedures to prevent hip dislocation in children with $\mathrm{CP}$ are performed in 7 university hospitals and 5 regional hospitals (10). Preventive surgery is recommended when the migration percentage (MP) (5) exceeds $40 \%$. However, there are no specific recommendations regarding when APT or VDRO should be performed, nor when VDRO should be combined with pelvic osteotomy or whether VDRO should be performed uni- or bilaterally in children with unilateral displacement.

This study included all children in CPUP in GMFCS levels II-V who underwent surgery with APL or VDRO before the age of 14 years and who had data reported for the range of hip abduction preoperatively and 6-18 and 18-36 months postoperatively. For children who underwent APL, only those who had this operation as their 1st hip operation were included. For children who underwent VDRO, those who were operated on with simultaneous APL and those who had been operated on with APL before the index operation were also included. Those operated on with a second operation before the 18-36month examination were excluded. Sex, GMFCS level, and age at the time of surgery were registered. For VDRO, we recorded whether the operation was performed uni- or bilaterally. Hips operated on with VDRO in combination with pelvic osteotomy were analyzed separately.

\section{Statistics}

The mean value, standard deviation (SD), and 95\% confidence interval (CI) were calculated for variables for all children. These values were obtained before and at 6-18 months and 18-36 months after the operation.
Number of children (\%) according to sex, GMFCS level, and type of operation

\begin{tabular}{lccc}
\hline Factor & $\begin{array}{c}\text { APL } \\
\mathrm{n}=150\end{array}$ & $\begin{array}{c}\text { VDRO } \\
\mathrm{n}=157\end{array}$ & $\begin{array}{c}\text { Total } \\
\mathrm{n}=307\end{array}$ \\
\hline Boys & $88(59)$ & $91(58)$ & 179 \\
Girls & $62(42)$ & $66(42)$ & 128 \\
GMFCS level & $4(4)$ & $3(2)$ & 7 \\
$\quad$ II & $17(11)$ & $12(8)$ & 29 \\
III & $55(37)$ & $34(22)$ & 89 \\
IV & $74(49)$ & $108(69)$ & 182 \\
V & & & \\
Mean age at time of the & $5.4(2.8)$ & $7.1(2.8)$ & \\
$\quad$ operation, years (SD) & $1.2-13.8$ & $2.2-13.8$ & \\
$\quad$ range & & & \\
\hline
\end{tabular}

In the assessment of the postoperative range of abduction, data from 1 hip per child was used. In children who underwent a bilateral operation, the hip with the lowest preoperative range of abduction was included. In those who underwent a bilateral operation but whose hips had symmetrical mobility preoperatively, the side included in the data analysis was randomized. In the analysis of the differences between sides, the difference between the 2 hips was calculated preoperatively and at 6-18 and 18-36 months postoperatively.

To adjust for possible confounders we used ordinary linear regression with the postoperative measurement as the dependent variable. As the independent variables, treatment indicator variables for APL versus VDRO, and the age at the time of the operation, sex, GMFCS level, and preoperative abduction level were used. Residuals were checked by plotting them against each covariate as well as in a Q-Q plot to ensure the model fit the data.

\section{Ethics, funding, and potential conflicts of interest}

The study was approved by the Medical Research Ethics Committee at Lund University (LU-443-99). The study was funded by Stiftelsen för bistånd åt rörelsehindrade i Skåne. The authors declare no conflict of interest.

\section{Results}

307 children (179 boys, 128 girls) who received APL ( $\mathrm{n}=$ $150)$ or VDRO $(\mathrm{n}=157)$ fulfilled the inclusion criteria and were included in this study. In addition to these, 23 children were excluded due to second surgery before the 18-36-month follow-up: 13 were primarily operated on with APL, 10 with VDRO. There mean range of abduction preoperatively was $29^{\circ}$ (CI 28-31). Children who underwent VDRO were more often in GMFCS level V, and their mean age at surgery was 1.7 years higher compared with children operated on with APL (Table). Of the 157 children who underwent VDRO, 64 also had a pelvic osteotomy. 
Mean $(95 \% \mathrm{Cl})$ hip abduction $\left({ }^{\circ}\right)$

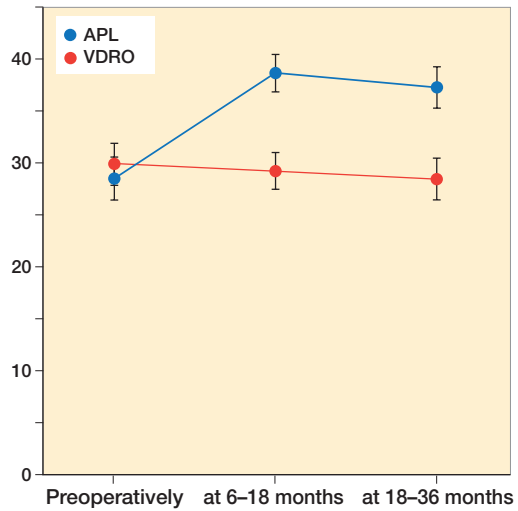

Figure 1. Mean range of hip abduction with $95 \%$ confidence intervals preoperatively and at 6-18 and 18-36 months after adductor-psoas lengthening (APL) and varus derotation osteotomy performed (VDRO).

In children who underwent APL, the mean range of abduction increased from $29^{\circ}$ (CI 27-31) preoperatively to $39^{\circ}$ (CI $37-40)$ at 6-18 months, and $37^{\circ}$ (CI 35-39) at 18-36 months postoperatively (Figure 1). In children who underwent VDRO, the respective values were $30^{\circ}$ (CI 28-32), $29^{\circ}$ (CI 28-31), and $29^{\circ}$ (CI 27-31) at the same follow-up intervals. In children who received the VDRO in combination with a pelvic osteotomy, the respective values were $28^{\circ}$ (CI 25-31), $27^{\circ}$ (CI 25-30), and $27^{\circ}$ (CI 24-30) at the same follow-up intervals. The mean change in range of abduction was $8.8^{\circ}$ (CI 6.8-11) after APL and $-1.4^{\circ}$ (CI -3.4 to 0.5 ) after VDRO at $18-36$ months. The unadjusted difference in mean change between APL and VDRO was $10^{\circ}$ (CI 7.4-13); the adjusted difference was $8.0^{\circ}$ (CI 5.4-11).

The absolute preoperative difference in hip abduction between sides was higher in children who received a unilateral VDRO $\left(9.4^{\circ}\right.$, CI 7.8-11) than in those who received a bilateral VDRO $\left(5.5^{\circ}, \mathrm{CI} 3.4-7.6\right)$ or bilateral APL $\left(6.1^{\circ}, \mathrm{CI}\right.$ 5.0-7.2) (Figure 2). After unilateral VDRO, the difference between sides increased marginally to $11^{\circ}(\mathrm{CI} 8.9-12)$ at 6-18 and $11^{\circ}(\mathrm{CI} 8.7-13)$ at $18-36$ months postoperatively. In children who received a bilateral VDRO, the difference decreased to $4.8^{\circ}$ (CI 2.8-6.6) at 6-18 months but then increased to $5.9^{\circ}$ (CI 3.7-8.1) at 18-36 months. In children who underwent a bilateral APL, the difference between sides decreased to 5.1 ${ }^{\circ}$ (CI 4.1-6.1) and then increased slightly to $5.4^{\circ}$ (CI 4.3-6.5) at 18-36 months. The crude changes in the difference between sides from the baseline to 18-36 months was for bilateral VDRO $1.1^{\circ}$ (CI -1.4 to 3.7 ), for unilateral VDRO $1.9^{\circ}$ (CI -0.5 to 4.2 ), and for APL $0.5^{\circ}$ (CI -1.0 to 2.0 ). Adjusted comparisons yielded $0.3^{\circ}$ (CI -2.0 to 2.5 ) difference for bilateral VDRO and $3.5^{\circ}$ (CI 1.4-5.7) for unilateral VDRO, respectively. Bootstrap standard errors were generated for all models of side differences, but results were similar (data not shown) and original results were retained.

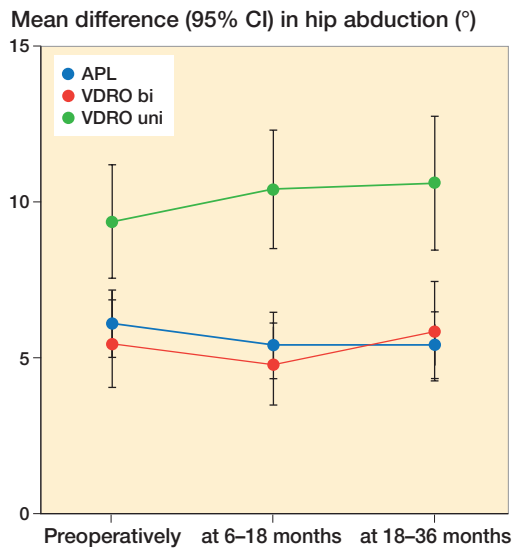

Figure 2. Mean difference in range of hip abduction between sides with $95 \%$ confidence intervals preoperatively and at 6-18 and 18-36 months after bilateral adductor-psoas lengthening (APL) and varus derotation osteotomy performed bilaterally (VDRO bi) and unilaterally (VDRO uni).

\section{Discussion}

The most important finding was that the mean range of hip abduction increased after APL but remained unchanged after VDRO. The change in mean difference in abduction between sides after VDRO was $<6^{\circ}$ at $18-36$ months after the operation, regardless of whether the operation was performed unior bilaterally.

The joint range of motion decreases gradually with age in children with CP. A previous study of the total population of children with $\mathrm{CP}$ in southern Sweden showed a reduced range of abduction from $43^{\circ}$ to $34^{\circ}$ between the ages of 2 and 14 years (2). From the age of 6 years, the magnitude of reduction did not differ between children in GMFCS levels IV and V in that study, which suggests that the differences in the distribution of GMFCS level and age between the children who received the APL or VDRO in our study did not affect the results. This was confirmed by the multiple regression analysis after adjustment for possible confounders.

After preventive surgery with APL, the mean lateral displacement as measured using Reimers' MP (5) decreases gradually or continues to increase at a slower velocity (15). This may reflect the increased ability to abduct postoperatively and the resultant altered force vector over the hip joint. A study of standing exercise after APL showed that children standing with $30^{\circ}$ abduction had a faster reduction in MP and a reduced recurrence rate compared with those standing with fewer degrees of abduction (16).

After reconstructive surgery with VDRO, there is an instantaneous reduction in MP. A study based on data from CPUP (15) showed that this immediate postoperative reduction is followed by a gradual increase in the mean MP. The unchanged range of abduction postoperatively indicates that VDRO does not affect the postoperative positioning of the hip, which leaves the force vector unchanged and may explain 
the increasing MP postoperatively. Shore et al. (12) found that VDRO combined with APL reduced the risk for recurrence, which could be explained by an increased capability for abduction and improved positioning postoperatively.

After both APL and VDRO, there is a substantial risk of recurrence and the need for repeated surgery $(9-12,17)$. Studies have shown that the strongest predictor of outcome after APL is a preoperative high degree of displacement $(\mathbf{1 1 , 1 7 )}$. This may be because the postoperative change in the force vector is not sufficient to reverse the development of lateralization in these hips. The strongest predictor of outcome after VDRO is young age at the time of the operation (12). The gradually increasing lateralization after VDRO means that children who are operated on at an early age have a longer period of development ahead of them and a longer period of time for the displacement to increase.

The postoperative difference in abduction was similar between sides after VDRO in our study, regardless of whether the operation was performed uni- or bilaterally. The maximum possible mean differences between uni- and bilateral in change from baseline to 18-36 months given the confidence intervals was $<6^{\circ}$.

Owers et al. (18) performed bilateral APL and VDRO on 30 children with the goal of creating symmetry between the hips. At the 3-year follow-up, 13 of the children had a windswept deformity, compared with 15 preoperatively. This finding suggests that the muscle imbalance that creates unilateral hip displacement is not permanently rebalanced by bilateral surgery.

Our study has some limitations. We did not have data on the degree of varization, amount of shortening, and soft-tissue release for children who received a VDRO. A strength of our study is that the data were obtained from the total population of children in Sweden with CP, reducing the risk of selection bias, and that the measurements were performed in a standardized way. Only 23 children were excluded due to early reoperation and this should not have affected the result.

In conclusion, the range of hip abduction increased after APL but remained unchanged after VDRO. This finding increases understanding of hip development after these operations. The difference in abduction between sides was not greatly affected by whether VDRO was performed uni- or bilaterally.

Study design: GH, PW. Data collection: GH. Statistical analysis: PW. Manuscript preparation: $\mathrm{GH}, \mathrm{PW}$. The study was reported according to STROBE guidelines.

Acta thanks Erich Rutz and Michael Sussman for help with peer review of this study.
1. Miller F, Slomczykowski M, Cope R, Lipton G E. Computer modeling of the pathomechanics of spastic hip dislocation in children. J Pediatr Orthop 1999; 19: 486-92.

2. Nordmark E, Hägglund G, Lauge-Pedersen H, Wagner P, Westbom L. Development of lower limb range of motion from early childhood to adolescence in cerebral palsy: a population based study. BMC Med 2009; 7: 65 .

3. Rang M, Silver R, de la Garza J. Cerebral palsy. In: Lovell W W, Winter R B, editors. Pediatric orthopaedics, 2nd ed. Philadelphia: J B Lippincott; 1986. p. 345-96.

4. Samilson R L, Carson J J, James P, Raney F L Jr. Results and complications of adductor tenotomy and obturator neurectomy in cerebral palsy. Clin Orthop Relat Res 1967; 54: 61-73.

5. Reimers J. The stability of the hip in children: a radiological study of the results of muscle surgery in cerebral palsy. Acta Orthop Scand Suppl 1980; 184: 1-100.

6. Silver R L, Rang M, Chan J, de la Garza J. Adductor release in nonambulant children with cerebral palsy. J Pediatr Orthop 1985; 5: 672-7.

7. Canavese F, Emara K, Sembrano J N, Bialik V, Aiona M D, Sussman M D. Varus derotation osteotomy for the treatment of hip subluxation and dislocation in GMFCS level III to V patients with unilateral hip involvement: follow-up at skeletal maturity. J Pediatr Orthop 2010; 30: 357-64.

8. Larsson, M, Hägglund G, Wagner P. Unilateral varus osteotomy of the proximal femur in children with cerebral palsy: a five-year follow-up of the development of both hips. J Child Orthop 2012; 6: 145-51.

9. Terjesen T. Femoral and pelvic osteotomies for severe hip displacement in nonambulatory children with cerebral palsy: a prospective populationbased study of 31 patients with 7 years' follow-up. Acta Orthop 2019; 90: 614-21.

10. Kiapekos N, Broström E, Hägglund G, Åstrand P. Primary surgery to prevent hip dislocation in children with cerebral palsy in Sweden: a 5-year follow-up by the national surveillance program (CPUP). Acta Orthop 2019; 90: 495-500.

11. Shore B J, Yu X, Desai S, Selber P, Wolfe R, Graham H K. Adductor surgery to prevent hip displacement in children with cerebral palsy: the predictive role of the Gross Motor Function Classification System. J Bone Joint Surg Am 2012; 94: 326-34.

12. Shore B J, Zurakowski D, Dufreny C, Powell D, Matheney T H, Snyder B D. Proximal femoral varus derotation osteotomy in children with cerebral palsy: the effect of age, Gross Motor Function Classification System level, and surgeon volume on surgical success. J Bone Joint Surg Am 2015; 97: 2024-31.

13. Alriksson-Schmidt A, Arner M, Westbom L, Krumlinde-Sundholm L, Nordmark E, Rodby-Bousquet E, et al. A combined surveillance program and quality registry improves management of childhood disability. Disabil Rehabil 2017; 39: 830-6.

14. Palisano R J, Rosenbaum P, Bartlett D, Livingston M H. Content validity of the expanded and revised Gross Motor Function Classification System. Dev Med Child Neurol 2008; 50: 744-50.

15. Wagner P, Hägglund G. Hip development after surgery to prevent hip dislocation in children with cerebral palsy: a longitudinal register study of 252 children. Acta Orthop 2021; accepted.

16. Martinsson C, Himmelmann K. Abducted standing in children with cerebral palsy: effects on hip development after 7 years. Pediatr Phys Ther 2021; 33: 101-7.

17. Terjesen T. To what extent can soft-tissue releases improve hip displacement in cerebral palsy? Acta Orthop 2017; 88: 695-700.

18. Owers K L, Pyman J, Gargan M F, Witherow P J, Portinaro N M. Bilateral hip surgery in severe cerebral palsy: a preliminary review. J Bone Joint Surg 2001; 83-B: 1161-7. 